INRA Prod. Anim., 2010, 23 (1), 205-214

\title{
Contaminants chimiques organiques des oufs de poule pondeuse : aspects réglementaires, modalités et risques de transfert
}

\author{
C. JONDREVILLEE,2, A. FOURNIER ${ }^{1,2,3}$, A. TRAVEL ${ }^{3}$, C. FEIDT'1,2, B. ROUDAUT 4 \\ ${ }^{1}$ INRA, USC340 Animal et Fonctionnalités des Produits Animaux, F-54505 Vandouvre-lès-Nancy, France \\ 2 Université Nancy, Animal et Fonctionnalités des Produits Animaux, F-54505 Vandouvre-lès-Nancy, France \\ 3 ITAVI, F-37380 Nouzilly, France \\ ${ }^{4}$ AFSSA, Laboratoire d'Etudes et de Recherches sur les Médicaments Vétérinaires et Désinfectants, \\ F-35133 Javené, France \\ Courriel : catherine.jondreville@ensaia-inpi-nancy.fr
}

\begin{abstract}
Certaines substances chimiques organiques ingérées de manière involontaire par les poules pondeuses (contaminants de l'environnement) ou introduites de manière volontaire dans leur alimentation (médicaments, additifs) sont susceptibles d'être transférées vers l'œuf. Des études de pharmacocinétique permettent de comprendre les modalités de ce transfert et fournissent les bases scientifiques de la réglementation sur les médicaments vétérinaires, les additifs et les aliments pour animaux. La présence de contaminants de l'environnement dans les œufs de poules élevées en plein air est difficile à maîtriser en raison de leur ingestion variable de matrices environnementales, notamment de sol.
\end{abstract}

Au cours de l'élevage, les animaux peuvent se trouver en contact avec des substances chimiques susceptibles d'être transférées vers les denrées alimentaires d'origine animale. Présentes dans les produits animaux, ces substances, ainsi que leurs produits de dégradation ou métabolites, représentent un danger potentiel pour la santé des consommateurs, et peuvent être à l'origine de difficultés technologiques de transformation. Ces composés chimiques peuvent être intentionnellement administrés aux animaux (médicaments vétérinaires, additifs alimentaires) ou administrés involontairement via l'alimentation, tels que des pesticides utilisés pour la production de matières premières. Les animaux peuvent également se trouver en contact avec des contaminants rémanents de l'environnement, tels que des pesticides organochlorés, ou d'autres composés organiques d'origine non agricole. Or, le consommateur exige un produit sain, exempt de contamination chimique qui pourrait nuire à sa santé. Cette demande est largement relayée par les autorités sanitaires et la réglementation et il est de la responsabilité de chaque éleveur d'assurer que les produits qu'il commercialise sont conformes aux niveaux de résidus édictés par la réglementation.
Le présent article fait le point sur les composés chimiques auxquels les poules pondeuses peuvent être exposées, de façon volontaire ou involontaire, au cours de leur élevage, en décrivant leur origine, ainsi que la réglementation qui encadre la fourniture de produits sains. Les principes scientifiques sur lesquels repose cette réglementation sont ensuite abordés. Les travaux scientifiques présentés permettent d'évaluer le risque de transfert des composés chimiques vers l'œuf, de comprendre les modalités de ce transfert et de mettre en évidence ses facteurs de variation, qu'ils soient liés aux caractéristiques des molécules ou aux performances des animaux. Des résultats d'enquêtes réglementaires ou non sont ensuite présentés et les sources de non-conformité analysées.

\section{1 / Contaminants chimiques dans les denrées alimentai- res d'origine animale : ori- gine et contexte réglemen- taire}

La réglementation diffère substantiellement suivant le type de composé auquel l'animal est exposé. Certains sont autorisés et leur usage est strictement réglementé. D'autres, au contraire, comme certains polluants organiques persistants, peuvent exister de façon rémanente ou accidentelle dans l'environnement d'élevage des animaux ou dans leur alimentation.

\section{1 / Substances réglementées}

Parmi les molécules réglementées figurent des substances phytopharmaceutiques utilisées lors de la production de matières premières destinées à 1'alimentation des animaux. Ces molécules ne sont pas introduites intentionnellement dans l'alimentation des animaux. Au contraire les médicaments vétérinaires et les additifs utilisés en alimentation animale sont prescrits et utilisés intentionnellement, selon des itinéraires strictement encadrés pour garantir leur sécurité et leur efficacité. Toutes ces substances sont évaluées en termes de risque avant d'être autorisées et mises à disposition sur le marché.

a) Notion de limite maximale de résidu

Un concept s'est imposé pour ces substances: les denrées alimentaires ne doivent pas contenir de résidus à des teneurs susceptibles de présenter un 
Tableau 1. Limites Maximales de Résidus (LMR) pour les médicaments vétérinaires dans les œufs.

\begin{tabular}{|l|l|l|c|l|}
\hline \multicolumn{1}{|c|}{ Famille } & \multicolumn{1}{|c|}{ Molécule } & \multicolumn{1}{|c|}{ Résidu marqueur } & LMR( $\boldsymbol{\mu g} / \mathbf{k g})$ & Espèces \\
\hline & Chlortétracycline & Chlortétracycline et épimère en 4 & 200 & Volailles \\
& Oxytétracycline & Oxytétracycline et épimère en 4 & 200 & Volailles \\
& Tétracycline & Tétracycline et épimère en 4 & 200 & Volailles \\
& Colistine & Colistine & 300 & Volailles \\
& Lincomycine & Lincomycine & 50 & Volailles \\
& Erythromycine & Erythromycine A & 150 & Volailles \\
& Tylosine & Tylosine & 200 & Volailles \\
& Néomycine & Néomycine B & 500 & Volailles \\
& Tiamuline & Tiamuline & 1000 & Poules \\
\hline \multirow{2}{*}{ Anthelmintiques } & Flubendazole & Flubendazole & 400 & Volailles \\
& Pipérazine & Pipérazine & 2000 & Poules \\
\hline Antiparasitaires & Phoxime & Phoxime & 60 & Poules \\
\hline externes & Lasalocide & Lasalocide & 150 & Volailles \\
\hline Anticoccidiens & &
\end{tabular}

risque pour la santé du consommateur. Est considéré comme «résidu» toute substance pharmacologiquement active présente dans les denrées alimentaires (viande, abats, lait, œuf et miel) issues d'animaux exposés à une préparation. Il peut s'agir du (des) principe(s) actif(s) de la préparation administrée, d'excipients, mais également de produits de dégradation ainsi que des métabolites de ces principes actifs. C'est le cas par exemple des nitrofuranes (substances interdites) pour lesquels le contrôle porte sur la recherche de métabolites liés aux protéines. L'évaluation des potentialités toxiques d'un résidu permet de déterminer la dose sans effet toxique observable chez l'animal (DES) et d'extrapoler la dose journalière admissible chez l'homme (DJA), en tenant compte de facteurs de sécurité. Ayant déterminé l'évolution des résidus par des études de métabolisme, il devient possible de définir le résidu marqueur et de calculer des limites maximales de résidus (LMR) qui permettent de ne pas dépasser la DJA. Pour les médicaments vétérinaires cette mâ̂trise du risque est fondée sur la notion de temps d'attente, spécifique de chaque médicament vétérinaire, pour éviter la présence de résidus à des concentrations supérieures aux LMR établies pour les substances actives.

\section{b) Fixation des limites maximales de} résidus

La directive 2001/82/CE institue un code communautaire relatif aux médicaments vétérinaires pouvant être autorisés chez les animaux producteurs d'aliments. Le règlement 2009/470/CE, qui remplace le règlement $\mathrm{CEE} 2377 / 90$ depuis le 6 mai 2009, établit la procédure évoquée ci-dessus pour la fixation des LMR dans les denrées d'origine animale et le classement dans une liste des principes actifs autorisés dans les médicaments vétérinaires. Cette évaluation est réalisée par l'Agence Européenne du Médicament (EMA)
Les médicaments vétérinaires pour lesquels aucune LMR ne peut être fixée sont interdits dans l'ensemble des pays membres de l'Union européenne : chloramphénicol, nitrofuranes et nitroimidazoles en font partie. Plusieurs familles de médicaments vétérinaires sont autorisées chez les volailles : antibiotiques, antiparasitaires, anthelminthiques, anticoccidiens. Cependant, le nombre de molécules disponibles est restreint chez les poules pondeuses en raison de la production d'œufs en continu et du risque de transfert des résidus dans l'œuf. Les traitements s'effectuent essentiellement par voie orale : aliment ou eau de boisson pendant 5 à 7 jours, excepté pour le traitement par des antiparasitaires externes, qui est réalisé par pulvérisation des surfaces qui entourent les animaux.

Neuf LMR ont été fixées dans les œufs, au niveau européen, pour les résidus d'antibiotiques (tableau 1) appartenant aux familles des tétracyclines, polypeptides, macrolides, aminosides et pleuromutilines. Aucun sulfamide, ni pénicilline, ni quinolone n'est autorisé chez les poules pondeuses en production. Les LMR ont également été établies pour trois antiparasitaires et un anticoccidien. Un anticoccidien peut aussi être utilisé par voie orale sans LMR : l'amprolium.

Les LMR concernent généralement la molécule mère. Les temps d'attente sont ensuite déterminés pour chaque spécialité vétérinaire de façon à ce que les LMR ne soient pas dépassées dans un tissu donné. Le respect des temps d'attente est particulièrement délicat chez les poules pondeuses dans la mesure où le producteur doit retirer du marché sa production d'œufs pendant le traitement et, dans certains cas, plusieurs jours après le traitement. Les molécules, qui ont obtenu une LMR pour les œufs, ne dépassent pas le plus souvent cette LMR pendant les traitements préconisés par le vétérinaire.
L'évaluation des additifs alimentaires est conduite selon le règlement 2003/1831/CE par l'autorité européenne de sécurité sanitaire des aliments (EFSA). Certains de ces additifs coccidiostatiques ne sont autorisés chez les poulettes destinées à la ponte que jusqu'à l'âge de 12 à 16 semaines : halofuginone, diclazuril, lasalocide, salinomycine et monensin. Depuis janvier 2006, tous les additifs antibiotiques sont interdits dans l'Union Européenne.

Les résidus de pesticides dans les denrées alimentaires d'origine animale sont régis par la directive 86/363/CEE et les LMR sont fixées dans le cadre du règlement 396/2005/CE. Elles sont établies par couple «matière activedenrée». Des études de transfert sont né-cessaires lorsque les traitements phytosanitaires conduisent à des teneurs excédant $0,1 \mathrm{mg}$ de résidu/ $\mathrm{kg}$ de plante destinée à l'alimentation des vaches laitières ou des poules pondeuses ou lorsque des études de métabolisme indiquent un risque de bioaccumulation. C'est le cas des pesticides lipophiles, présentant un log Kow (coefficient de partition octanol/eau) supérieur à 3 .

\section{2 / Les contaminants non intentionnels}

Des contaminants tels que les polluants organiques persistants (POP) peuvent se trouver dans l'environnement des animaux ou dans leur alimentation. Les POP sont des substances organiques qui associent des propriétés leur conférant un caractère toxique, une bioaccumulation potentielle, une stabilité dans les milieux et une propension à migrer vers des milieux éloignés, notamment par voie atmosphérique. Ces molécules sont considérées comme préoccupantes par la réglementation internationale qui a dressé la liste nominative de 12 substances dans le cadre de la convention de Stockholm signée en mai 2001. Parmi ces molécules figurent des pesticides (DDT, endrine, 
aldrine, dieldrine, hexachlorobenzène...), des produits industriels comme les polychlorobiphényles (PCB), mais également des substances involontairement produites lors de processus de combustion incomplète de matière organique, comme les dioxines (PCDD) et les furanes (PCDF), qui proviennent principalement de l'incinération des déchets, et les hydrocarbures aromatiques polycycliques (HAP), qui sont principalement issus de la combustion en zone urbaine et du transport routier. Neuf produits chimiques supplémentaires, pesticides (lindane, chlordécone...) ou retardateurs de flamme bromés (certains polybromodiphényléthers, PBDE), ont récemment été ajoutés à cette liste.

La réglementation européenne fixe des teneurs maximales dans les denrées alimentaires de certains de ces contaminants (hors pesticides, Règlement $\mathrm{CE}$ 1881/2006). La réglementation européenne impose un maximum de 3 pg OMSTEQ PCDD/F/g de matière grasse (MG) dans les œufs et les ovoproduits. Ces valeurs sont doublées lorsque les PCB dioxin-like (PCB DL) sont inclus. $\mathrm{La}$ fixation d'un maximum légal de $50 \mathrm{ng} / \mathrm{g}$ MG pour les PCB non dioxin like (PCB NDL) est en cours de discussion. Les valeurs maximales réglementaires sont de $0,01 \mathrm{mg}$ de lindane, $0,05 \mathrm{mg}$ de DDT et $0,02 \mathrm{mg}$ aldrine + dieldrine par $\mathrm{kg}$ d'œuf. Cependant, ces maxima dans l'œuf ne sont pas établis pour les HAP ou dans les denrées alimentaires d'origine animale pour les PBDE. La limitation des niveaux de substances indésirables dans les matières premières et aliments destinés à l'alimentation des animaux (Directive 2002/32/CE) contribue à la prévention des risques liés à la contamination des produits animaux par des substances comme les POP et les métaux lourds. Cependant, ces contaminants peuvent être prélevés par les animaux directement dans leur environnement via l'ingestion de sol, de végétaux, de pédofaune ou de résidus de matériaux de construction.

\section{2 / Modalités de transfert vers l'œuf}

La connaissance des modalités et cinétiques de transfert, au sein de l'animal, des composés auxquels il est exposé, est indispensable à l'évaluation du risque de présence de ces composés dans les œufs. La voie d'exposition majeure est la voie orale, y compris pour les médicaments vétérinaires. Ces derniers sont pour la plupart destinés à agir de façon systémique, excepté certaines molécules qui agissent directement au niveau du tractus gastro-intestinal, les coccidiostatiques et (ou) qui sont peu absorbées, la colistine.

\section{1 / Schéma général de transfert à l'échelle de l'animal}

La pharmacocinétique ou toxicocinétique, qui se réfère à l'absorption, la distribution, le métabolisme et l'excrétion (ADME) d'un composé chimique (Renwick 2001) permet de décrire et de quantifier le devenir du composé dans l'organisme. Cette discipline permet en outre de déterminer le temps d'attente requis pour prévenir la présence de résidus de substances indésirables dans les tissus destinés à la consommation.

Après absorption au niveau intestinal, les molécules sont dirigées vers le foie. Le premier site de métabolisation est l'entérocyte au niveau du duodénum (Brugère 1992), mais la majorité des transformations (Anadon et al 1993) a lieu au niveau du foie (réactions de phase I : oxydation, réduction, hydrolyse et de phase II : conjugaison). Ces réactions sont une voie de défense de l'organisme, qui aboutit à la transformation d'une partie du xénobiotique ingéré en une forme qui pourra être éliminée, par exemple via l'urine ou via la bile. Après avoir atteint la circulation générale, les molécules sont distribuées dans les différents tissus et éliminées. L'œuf représente une des voies d'excrétion des médicaments et xénobiotiques chez la poule pondeuse. De nombreux facteurs liés aux caractéristiques de la molécule ingérée, tels que sa lipophilie, son caractère polaire, son aptitude à se lier aux protéines ou sa sensibilité à la métabolisation sont des éléments déterminants de ses taux et cinétique de transfert vers l'œuf. De plus, certains facteurs liés à l'animal peuvent constituer des éléments majeurs de modulation du transfert des xénobiotiques vers l'œuf.

\section{2 / Distribution des contami- nants et médicaments dans les constituants de l'œuf}

Les molécules lipophiles, comme le chloramphénicol (Samouris et al 1998) ou les POP, sont préférentiellement transférées vers le vitellus, riche en lipides. Au contraire, les acides faibles (sulfamides, quinolones), qui ont dans l'organisme une distribution extracellulaire, se concentrent dans l'albumen (Roudaut 1997) qui est un milieu polaire, composé d'eau à $88 \%$. La répartition des antibiotiques entre les deux constituants de l'œuf est très variable, allant de 23 à $95 \%$ pour l'albumen. Le tableau 2 indique pour différentes familles de médicaments vétérinaires, les rapports de concentrations entre l'albumen et le vitellus.

\section{3 / Cinétiques de transfert des contaminants et médicaments}

Après ingestion, les résidus apparaissent rapidement dans l'albumen (24 h après le début de l'administration), plus tardivement dans le vitellus (24 à $48 \mathrm{~h}$ après le début de l'administration). En effet, au moment de la première ingestion de xénobiotique ou de médicament, le jaune de l'œuf qui sera pondu le lendemain a déjà terminé sa phase de croissance et n'est donc pas exposé au contaminant ; au contraire l'albumen, déposé durant les 24 dernières heures précédant la ponte, est susceptible de contenir des résidus.

Pour les molécules fortement métabolisées ou peu stockées, comme c'est le cas pour la plupart des médicaments vétérinaires, le flux de résidus dans le jaune est proportionnel au dépôt de jaune (Donoghue et al 1997). Ce type de modèle peut également être appliqué à des contaminants lipophiles mais très sensibles à la métabolisation comme le benzo[a]pyrène (Fournier et al 2010).

Compte tenu du mode de formation et de dépôt du vitellus par accrétion, qui prend huit jours environ, le plateau n'est atteint qu'au bout d'une dizaine de jours (figure 1). La décontamination de l'œuf est également un processus qui n'excède pas le temps de formation du jaune, avec des cinétiques d'élimination le plus souvent rapides. En revanche, pour

Tableau 2. Répartition des résidus de médicaments dans l'œuf (d'après Kan et Petz 2000).

\begin{tabular}{|l|c|c|}
\hline \multicolumn{1}{|c|}{ Molécule } & pKa & Rapport albumen/vitellus \\
\hline Sulfamides & $5,5-7,5$ & $2-4$ \\
\hline Quinolones & $6,2-6,3$ & $0,4-9,6$ \\
\hline Tétracyclines & $3,3-8,3$ & $0,2-3$ \\
\hline Macrolides & $7,1-8,7$ & $0,3-1,4$ \\
\hline Aminosides & $>10$ & 0,05 \\
\hline Chloramphénicol & neutre & 0,3 \\
\hline Nitrofuranes & $7,2-9,3$ & $0,5-0,8$ \\
\hline Nitroïmidazoles & & 0,8 \\
\hline lonophores & & $0,04-1,25$ \\
\hline Nicarbazine (DNC) & & 0,007 \\
\hline Flubendazole & & 0,14 \\
\hline
\end{tabular}


Figure 1. Cinétique de transfert de deux pesticides lipophiles dans l'œuf de poule pondeuse (d'après MacLachlan 2008).

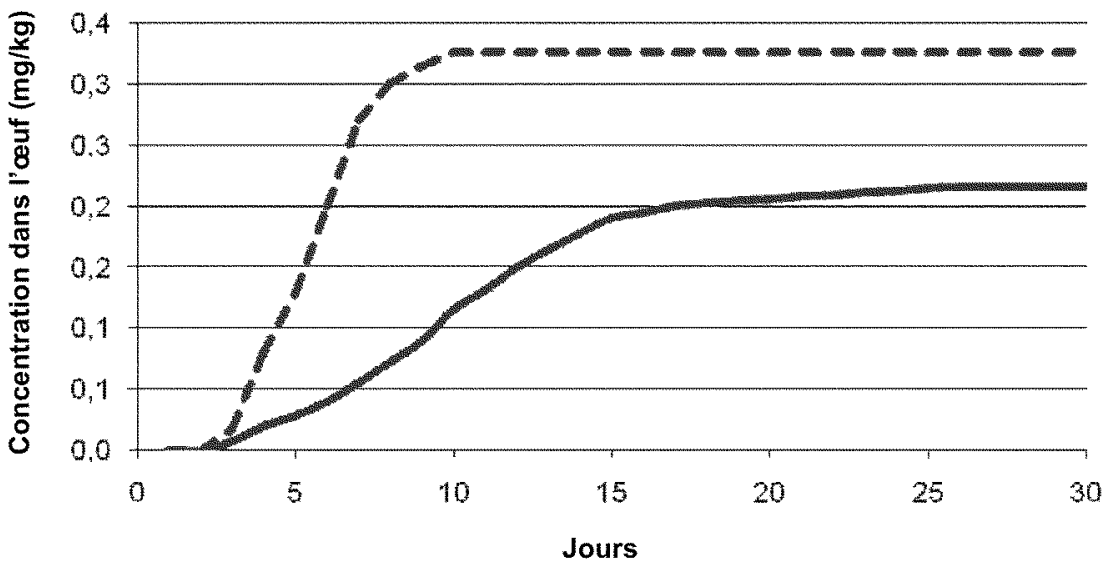

Lindare - - Difluberzuron

Estimations chez des poules pondeuses (poids corporel, 1,6 kg ; gras corporel, $256 \mathrm{~g}$; poids de l'œuf, $55 \mathrm{~g}$; consommation d'aliment, $104 \mathrm{~g} / \mathrm{j}$; taux de ponte, 0,85 ) consommant pendant 28 jours un aliment contenant 7,7 mg de diflubenzuron ( $\log \mathrm{Kow}=3,89$ ) et $1,5 \mathrm{mg}$ de lindane $(\log \mathrm{Kow}=3,78) / \mathrm{kg}$.

les molécules accumulées de façon significative, comme certains contaminants lipophiles peu sensibles à la métabolisation ou médicaments lipophiles (chloramphénicol), les flux de jaune et de contaminants ne sont pas proportionnels. Le temps nécessaire pour atteindre l'état d'équilibre, de même que le temps nécessaire pour retrouver un niveau de contamination basal de l'œuf après l'arrêt de l'exposition sont d'autant plus longs (Arnold et Somogyi 1986, MacLachlan 2008). Les modèles utilisés pour prédire le transfert de ce type de composés sont plus complexes et font intervenir des flux de stockage et de déstockage dans le tissu adipeux (Van Eijkeren et al 2006). A titre d'exemple, la figure 1 montre que le plateau est atteint au bout de 10 jours pour le diflubenzuron, sensible à la métabolisation, alors qu'il n'est atteint qu'au bout de 25 jours pour le lindane.

Pour les molécules lipophiles et peu métabolisées, les cinétiques et taux de transfert dépendent des performances, essentiellement du taux de ponte et de la composition corporelle des animaux. Par exemple, les concentrations de diflubenzuron dans l'œuf à l'équilibre seraient plus élevées et le temps de demi-vie plus long chez des White Leghorn que chez des poules produisant des œufs bruns comme les Brown Warren (Opdycke et Menzer 1984, MacLachlan 2008). Cette différence est attribuée à un compartiment gras périphérique plus important chez ces dernières.

Par ailleurs, dans le cas d'une exposition chronique à un contaminant lipophile persistant, la détérioration des performances de ponte, qui en limite l'excrétion, favorise son accumulation dans le gras corporel. La figure 2 est une simulation, chez des poules soumises à une exposition chronique à des $\mathrm{PCB}$, de l'effet de l'interruption de la ponte sur la concentration de PCB dans l'œuf. Lors de la première période, les teneurs en PCB de l'œuf et du gras abdominal augmentent pour atteindre

Figure 2. Simulation des teneurs en PCB non dioxin like (NDL) dans l'œuf et le gras abdominal chez des poules pondeuses ingérant quotidiennement $450 \mathrm{ng}$ de PCB NDL et présentant un arrêt de ponte, sans modification du gras corporel (d'après Fournier et al 2009).

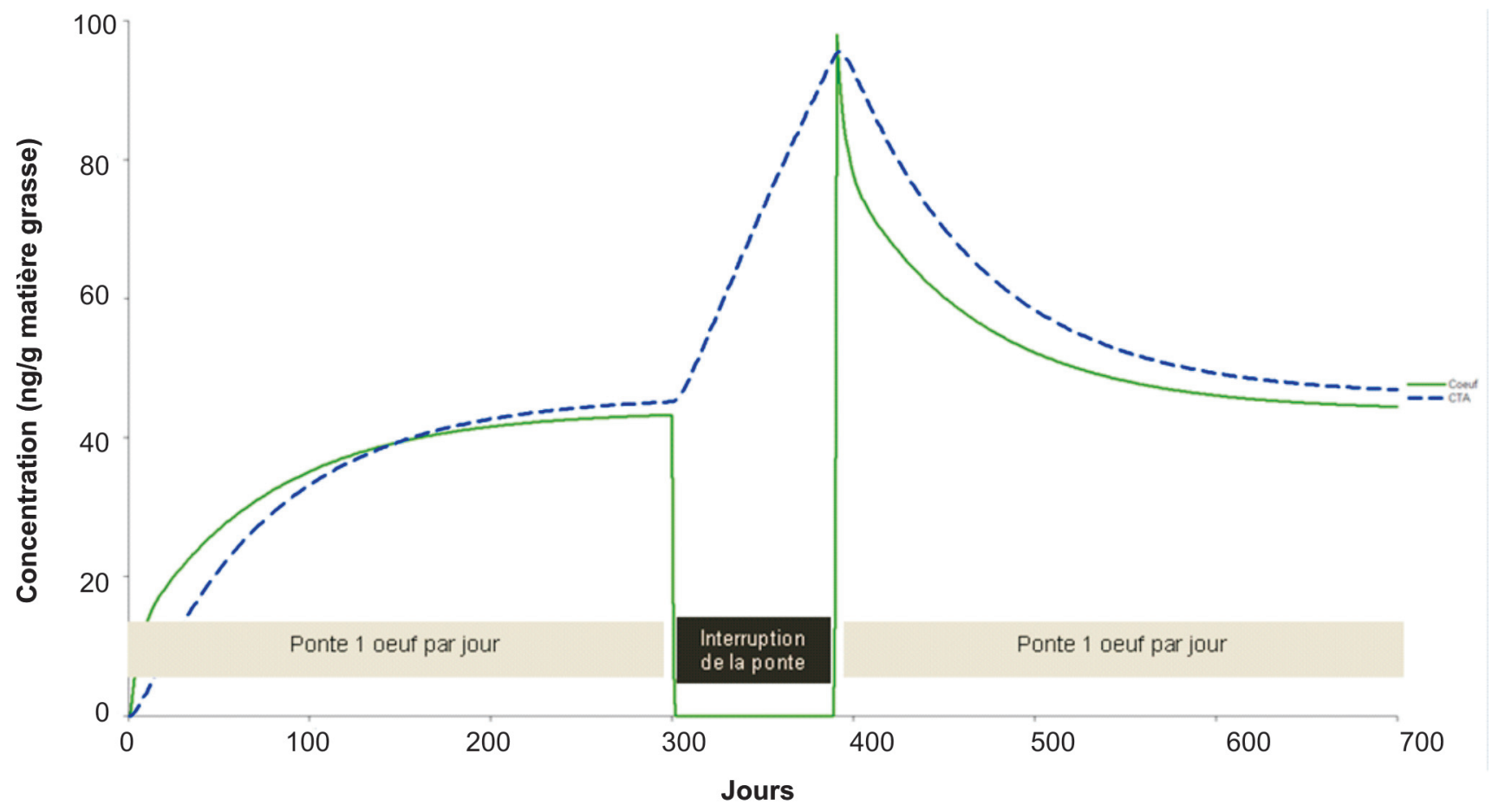


un état d'équilibre aux alentours de 250 jours. A ce moment, l'œuf et le gras corporel présentent les mêmes concentrations de $\mathrm{PCB}$, sur la base de la matière grasse. L'interruption de la ponte supprime l'évacuation des PCB via l'œuf et conduit à leur accumulation dans le gras corporel. Lors de la reprise de la ponte, compte tenu de cette nouvelle charge corporelle, la teneur dans les œufs est accrue et décroît progressivement vers l'état d'équilibre.

\section{4 / Facteurs de transfert et de bioaccumulation}

Ces études pharmacocinétiques sont indispensables au calcul des grandeurs nécessaires à l'estimation du risque de contamination des produits animaux par les xénobiotiques ou médicaments, tels que le taux de transfert ou le facteur de bioconcentration. Leur surestimation ou leur sous-estimation peuvent conduire à des surcoûts liés à des précautions trop drastiques ou au contraire à des risques pour la santé humaine.

Le taux de transfert des antibiotiques dans les œufs, après administration par voie orale (Roudaut 1997), est généralement faible $(<1 \%)$, voire très faible $(<0,01 \%)$ pour l'ampicilline et la tylosine. En revanche, pour les sulfamides et les quinolones (Roudaut 1998, Roudaut et Garnier 2002), le taux de transfert avoisine $1,5 \%$.

Pour les polluants organiques persistants, les taux de transfert excèdent souvent les $30 \%$ et les facteurs de bioconcentration excèdent la valeur 1 (tableau 3). Selon Leeman et al (2007) les pesticides anciens (les plus persistants) présentent un facteur de bioconcentration d'environ 1,4 contre 0,01 pour les pesticides actuellement autorisés. Pour les médicaments vétérinaires, cette valeur serait d'environ 0,03 . Pour les composés présentés en mélanges de congénères ( $\mathrm{PCB}$, dioxines/furanes), on estime que, selon le congénère, 5 à $50 \%$ des dioxines, furanes et $\mathrm{PCB}$ ingérés sont transférés vers l'œuf, 7 à $54 \%$ sont stockés dans le gras corporel et moins de 1\% dans le foie (Stephens et al 1995, Kan et Meijer 2007). Souvent, la valeur standard de $25 \%$ d'excrétion via les œufs est utilisée. Cependant, ces valeurs sont probablement sous-estimées car les durées d'expérimentation sont souvent inférieures au délai requis pour atteindre l'état d'équilibre. Le transfert des $\mathrm{PCDD} / \mathrm{F}$ et de la plupart des PCB vers l'œuf diminue lorsque le degré de chloration augmente (ou lorsque log Kow augmente) (figure 3). Les facteurs de transfert et de bioconcentration sont alors dépendants de la proportion de composés peu chlorés dans le mélange auquel les animaux sont exposés.

Tableau 3. Taux de transfert ${ }^{1}$ et facteur de bioconcentration ${ }^{2}$ de quelques polluants organiques persistants (d'après Kan et Meijer 2007, Leeman et al 2007, MacLachlan 2008).

\begin{tabular}{|l|c|c|c|}
\hline \multicolumn{1}{|c|}{ Composé } & $\begin{array}{c}\text { Demi-vie } \\
\text { (jours) }\end{array}$ & $\begin{array}{c}\text { Taux de } \\
\text { transfert }\end{array}$ & $\begin{array}{c}\text { Facteur de } \\
\text { bioconcentration }\end{array}$ \\
\hline DDT/DDE & 50 à 70 & 50 & 1,0 à 1,6 \\
Lindane & 5 à 14 & 10 & $0,13-0,2$ \\
Aldrine + dieldrine & 42 & 60 & 1,5 à 1,5 \\
Hexachlorobenzène & 50 à 60 & 55 & 1,5 à 1,9 \\
PCB & 30 à 100 & 5 à 90 & 0,09 à 2,2 \\
Heptachlore & 35 à 45 & 35 & 0,5 à 0,7 \\
PCDD/F & & 5 à 48 & 0,2 à 1,0 \\
\hline
\end{tabular}

1 Taux de transfert : rapport entre la quantité excrétée dans le produit par jour et la quantité quotidienne ingérée (exprimé en pourcentage).

2 Facteur de bioconcentration : rapport entre la teneur dans l'œuf (ng/g) et la teneur dans l'aliment $(\mathrm{ng} / \mathrm{g})$.

Figure 3. Relation entre l'hydrophobicité (Log Kow) et le facteur de bioconcentration (BCF) des PCDD/F du sol (ng/g) dans l'œuf (ng/g lipides) (d'après Stephens et al 1995 et Schuler et al 1997).

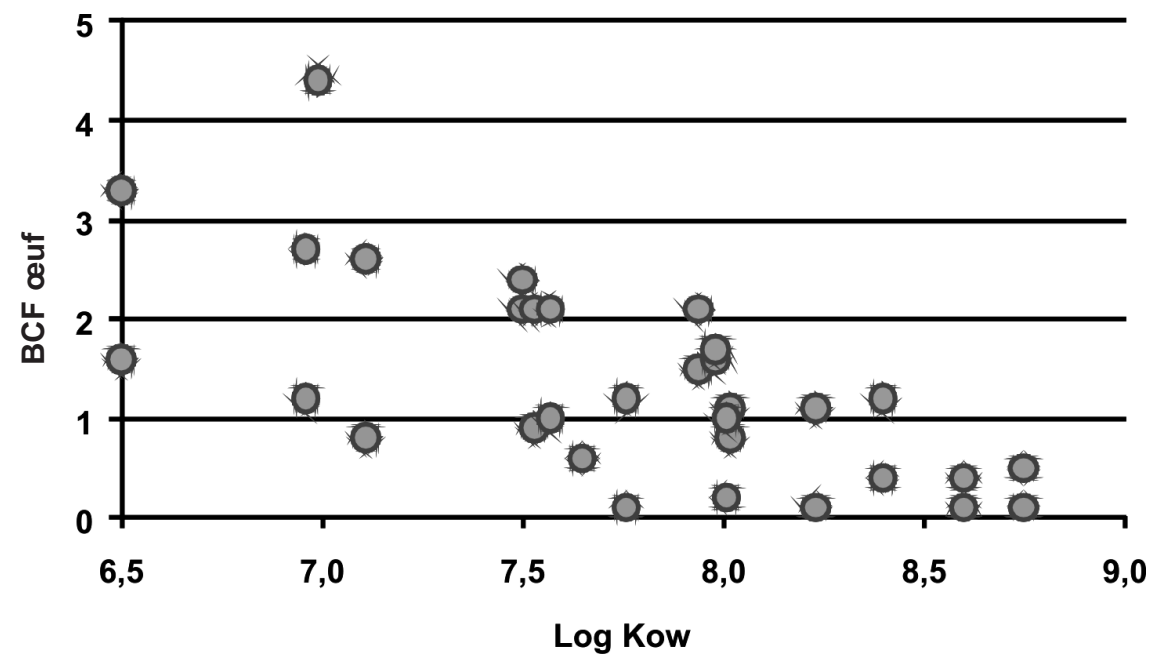

\section{3 / Stratégies de contrôle}

\section{1 / Contexte réglementaire}

Les contrôles officiels sont réalisés conformément à divers textes réglementaires d'origine communautaire, parmi lesquels figurent :

La directive 96/23/CE, concernant la recherche des résidus de composés chimiques dans l'ensemble des denrées alimentaires d'origine animale. Elle codifie :

- les plans de surveillance nationaux, avec le rôle des laboratoires nationaux de référence (LNR), et des laboratoires communautaires de références (LCR) ;

- la liste des groupes de contaminants à rechercher et les types de produits soumis à l'analyse (annexe I et II) : il s'agit des substances ayant un effet anabolisant et autres substances interdites, des médicaments vétérinaires et des contaminants de l'environnement à rechercher dans les viandes d'animaux de boucherie, les volailles, les produits de l'aquaculture, le lait, les œufs, les viandes de lapin et de gibier, et le miel ;

- la stratégie et la réalisation des échantillonnages (annexes III et IV, complétées par la décision 97/747/CE);

- Les missions et conditions de fonctionnement des laboratoires communautaires de référence (LCR) (annexe V).

La décision 2002/657/CE, qui concerne les performances des méthodes d'analyse et l'interprétation des résultats, dans le cadre de la mise en œuvre de la directive 96/23/CEE. Ce texte tient compte de l'évolution des techniques analytiques et précise les critères de performances et autres exigences que doivent remplir les méthodes 
d'analyse de confirmation, en distinguant la recherche des substances autorisées (avec LMR) et des substances interdites.

Cette législation pour une gestion sur le terrain du risque lié à ces substances conduit à la mise en œuvre de contrôles des résidus par les administrations compétentes : la DGAL au Ministère de l'Agriculture pour ce qui concerne les denrées alimentaires d'origine animale et la DGCCRF au ministère de l'Economie pour ce qui concerne les aliments pour animaux et les denrées transformées. Ces services s'appuient, par l'intermédiaire des Directions Départementales des Services Vétérinaires (DDSV), et depuis le $1^{\mathrm{er}}$ janvier 2010, par l'intermédiaire des Directions Départementales de la Protection des Populations (DDPP) sur un réseau de près de 90 laboratoires départementaux d'analyse et sur 11 laboratoires du Service Commun des Laboratoires (SCL). Les DDPP effectuent les contrôles tout au long de la chaîne alimentaire, incluant l'élevage, l'abattoir, l'importation, pour la mise en œuvre de plans de contrôle selon la directive 96/23/CE, destinés à apprécier les niveaux de contamination dans les diverses denrées alimentaires d'origine animale et à détecter d'éventuelles non-conformité là où le risque est le plus grand. Le nombre de prélèvements est établi conformément à l'annexe IV de la directive 96/23/CE ainsi qu'à la décision 97/747/CE. Ces textes communautaires précisent, pour chaque filière, le nombre de prélèvements à effectuer en fonction de la production nationale et en fonction des groupes de résidus à rechercher. Pour les volailles, le nombre annuel minimal d'animaux à contrôler est de un pour 200 tonnes de production avec un minimum de 100 échantillons par famille si la production est supérieure à 5000 tonnes.

\section{2 / Méthodes d'analyse}

La directive 96/23/CE prévoit le contrôle de l'ensemble des classes thérapeutiques utilisées en médecine vétérinaire. En France, des plans de contrôle ont été mis en place pour l'ensemble de ces classes : antibactériens, anthelminthiques, anticoccidiens ainsi que pour les pesticides organochlorés et les PCB.

La chromatographie liquide associée à la spectrométrie de masse en tandem est devenue la méthode de choix pour l'identification des substances interdites chez les animaux de rente. Cette méthode est applicable à diverses matrices, dont les œufs. Des contrôles sont aussi réalisés sur l'aliment ou l'eau de boisson destinés aux animaux.
Pour les substances autorisées, diverses techniques sont mises en œuvre impliquant le plus souvent une méthode de dépistage et une méthode de confirmation. Elles ciblent une ou des molécule(s) appartenant à une ou plusieurs familles et permettent de doser et d'identifier ces molécules sur la base de critères d'identification propres à la technique mise en œuvre.

\section{3 / Résultats des plans de contrôle}

Le nombre de prélèvements d'œufs de poule et de caille prévus en France en 2007 était de 1065 (Blanc-Gonnet et al 2009) et de 13685 pour l'Union Européenne. La matrice la plus fréquemment contrôlée est l'œuf entier. Le contrôle des substances interdites représente $42 \%$ des échantillons prélevés. Le chloramphénicol et les nitrofuranes ne sont plus détectés depuis plusieurs années. Pour les nitroimidazoles, seul un œuf a été trouvé positif en 2004 , soit un taux de contamination de $0,5 \%$.

Pour les antibiotiques, les molécules les plus fréquemment retrouvées sont les sulfamides, les tétracyclines et les quinolones. Les tétracyclines représentent $61 \%$ du tonnage d'antibiotiques utilisés dans la filière aviaire, ensuite viennent les sulfamides et les macrolides. Le taux de non-conformité le plus important concerne les anticoccidiens (BlancGonnet et al 2009) et plus particulièrement les additifs coccidiostatiques, avec environ $25 \%$ de non-conformité pour 2006 et 2007 . Aucune non-conformité relative aux contaminants de l'environnement n'a été relevée en France en 2007 dans le cadre de ce plan de contrôle.

\section{4 / Origine des non-confor- mité et prévention du risque en élevage}

\section{1 / Médicaments vétérinaires et additifs}

L'origine de non-conformité pour les additifs est essentiellement provoquée par des contaminations croisées, à différents stades de la filière, entre aliments blancs et aliments contenant des additifs (Cannavan et al 2000, Mortier et al 2005). La nicarbazine, très électrostatique, est la molécule la plus souvent mise en évidence (Dubreil et Chéneau 2009). La fixation de limites de tolérance dans les œufs (Règlement 2009/124/CE), depuis juillet 2009, devrait diminuer le taux de non-conformité pour ces molécules. Pour les médicaments vétérinaires, les principales causes de non-conformité sont l'utilisa- tion intentionnelle ou non d'un médicament non autorisé chez les poules pondeuses, le non respect de l'âge maximal d'administration, le non respect du temps d'attente ou de la posologie et la contamination croisée par des aliments supplémentés lors de la préparation de ces aliments à l'usine ou chez l'éleveur (Cannavan et al 2000). Une autre source de contamination, plus rare, est le recyclage des médicaments par ingestion de litière par les poules élevées sur litière (Kan 2005).

Le respect par l'éleveur des prescriptions de son vétérinaire et la prise en compte par celui-ci du risque «résidu» doivent permettre de garantir des aliments respectant les LMR. La démarche de certification des filières de production associée à la gestion des informations disponibles sur l'usage des médicaments (registre d'élevage) devrait permettre d'assurer la traçabilité des produits et des animaux traités, de réduire les risques de présence de résidus et de garantir les produits vis-àvis de ce risque.

\section{2 / Contaminants de l'environ- nement}

Des dépassements importants des limites maximales réglementaires de $\mathrm{PCB}$ et $\mathrm{PCDD} / \mathrm{F}$ ont été relevés au moment de la crise belge en 1999 avec des teneurs dans les œufs atteignant $46 \mu \mathrm{g}$ PCB indicateurs/g MG et de $32 \mathrm{pg}$ I-TEQ PCDD/F/g MG (Schoeters et Hogenboom 2006) ou lors de l'utilisation d'argiles contaminées comme antimottant dans les aliments (Schmid et al 2002). En dehors de ces situations accidentelles, des œufs issus de poules élevées en bâtiment, sans contact avec le milieu extérieur, contiennent 1 à 22 ng I-TEQ PCB DL/g MG et moins de $1 \mathrm{pg}$ I-TEQ PCDD/F/g MG (EFSA 2005, Thébault 2005, Travel et al 2009). Ces données révèlent l'efficacité des réglementations sur l'aliment qui limitent le transfert de ces contaminants vers les produits alimentaires.

Cependant, des enquêtes menées dans différents pays européens (Belgique, Suisse, Allemagne, Irlande, France, Suède, Royaume-Uni) révèlent la possible contamination par ces composés d'œufs de poules ayant accès à l'extérieur (Schoeters et Hogenboom 2006). L'ingestion de sol, végétaux et pédofaune est avancée comme la principale source de contaminants de l'environnement. Sur des sols clairement contaminés, en zone urbaine ou industrielle, des œufs excédant largement les 3 pg I-TEQ $\mathrm{PCDD} / \mathrm{F} / \mathrm{g} \quad \mathrm{MG}$ ont été collectés (Schuler et al 1997, Harnly et al 2000, Pussemier et al 2004, Kan 2005). Thébault (2005) rapporte des teneurs 
allant de 3 à 122 pg I-TEQ PCDD/F/g MG pour des œufs issus d'élevages de particuliers dans des zones réputées contaminées par un incinérateur. De même, des enquêtes portant sur des élevages de type biologique menées aux Pays-Bas et en Belgique ont montré que $13 \%$ des œufs collectés dans $26 \%$ des élevages enquêtés dépassaient la limite réglementaire de $3 \mathrm{pg}$ I-TEQ PCDD/F/g MG, suggérant une possible contamination y compris dans des zones rurales, a priori assez peu contaminées (De Vries et al 2006). Plus récemment, des enquêtes menées dans des élevages familiaux en Belgique révèlent la fragilité de ces œufs vis-à-vis des polluants de l'environnement, qu'il s'agisse de métaux lourds, comme le plomb, de PCB, dioxines-furanes ou pesticides organochlorés (Van Overmeire et al 2009). La même observation a été faite par Travel et al (2008), avec des teneurs en PCB, $\mathrm{PCDD} / \mathrm{F}$ et PBDE de l'ordre de 5 fois supérieures dans les élevages familiaux par rapport aux élevages professionnels.

S'agissant de contaminants provenant du sol, deux éléments sont à prendre en compte pour l'évaluation du risque : la quantité de contaminant ingérée, qui est la résultante de la teneur en contaminant du sol et de la quantité de sol ingéré, et la disponibilité du contaminant présent dans le sol.

\section{a) Quantité de sol ingérée}

Dans les élevages alternatifs, le parcours mis à disposition des animaux permet l'expression d'un comportement inné de recherche de nourriture à l'extérieur. Les intérêts sont multiples avec, outre la possibilité pour les animaux d'exprimer leur comportement naturel d'exploration et de recherche de nourriture, la possible diminution des coûts alimentaires et la limitation de l'importation d'éléments à risque $(\mathrm{N}, \mathrm{P}$, éléments trace métalliques) à l'échelle de l'élevage, grâce à la valorisation des ressources locales présentes sur le parcours. On estime qu'une poule sur parcours consomme $10 \mathrm{~g}$ de sol sec, $7 \mathrm{~g}$ de végétaux secs et $20 \mathrm{~g}$ d'insectes et lombrics par jour (De Vries et al 2006). Cependant, ces quantités sont sujettes à de larges variations. Des poules ayant un accès à l'extérieur ingèrent notamment des végétaux, même lorsqu'on leur apporte une alimentation équilibrée (Horsted et al 2007) (figure 4). Lorsque l'alimentation est déséquilibrée ou présente une granulométrie très grossière (blé entier + grit + coquille d'huîtres), le volume de matrice environnementale, notamment de sol, ingéré augmente. Ces auteurs ont aussi montré que les caractéristiques de la végétation présente sur le parcours influencent le comportement alimentaire des poules. La teneur en cendres des fientes, qui est un indicateur de l'ingestion de sol, atteint 25 à $80 \%$ chez des poules de particuliers représentant une ingestion de sol pouvant varier de 2 à $30 \mathrm{~g}$ par jour (Waegeneers et al 2009). Dans une étude menée dans notre laboratoire, les fientes contenaient 40 à $60 \%$ de cendres selon que les animaux recevaient un aliment standard ou aliment déséquilibré, à base de blé et de calcicoque uniquement. La limitation du risque de transfert des contaminants de l'environnement vers l'œuf nécessite donc de

Figure 4. Matrices présentes dans le proventricule de poules suivant les espèces végétales présentes sur le parcours et le type d'aliment ${ }^{1}$ distribué (d'après Horsted et al 2007).

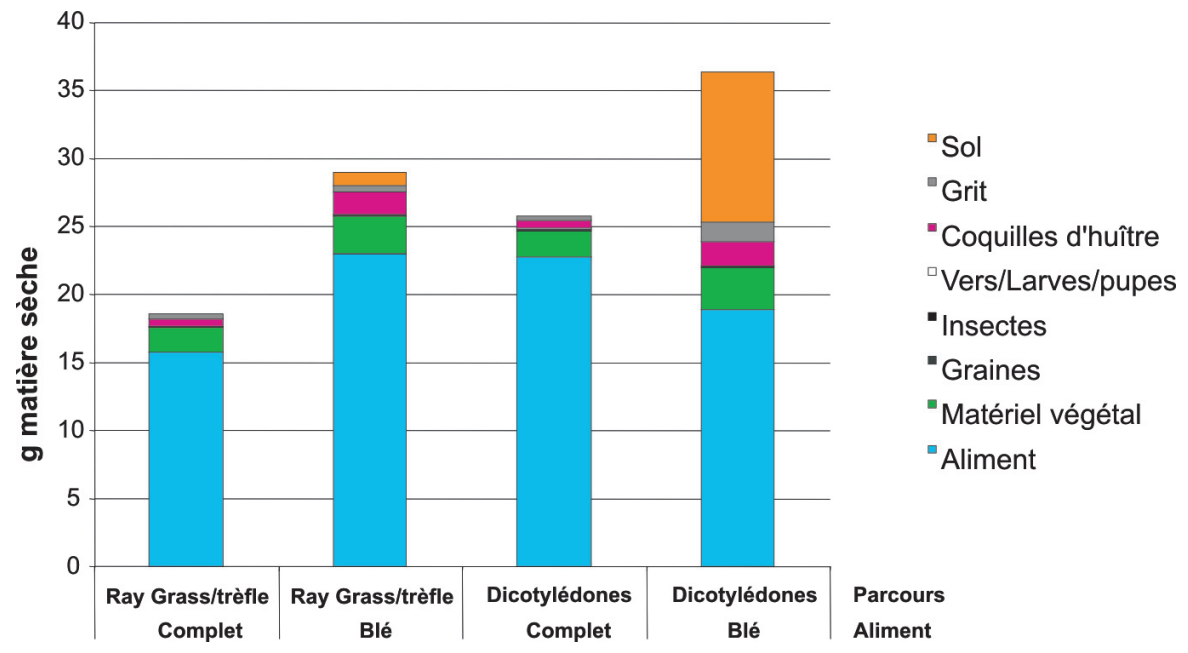

1 L'aliment est distribué à l'extérieur : il est composé d'un aliment complet + grit + coquilles d'huître ou de blé + grit + coquilles d'huître. connaître et d'évaluer les pratiques d'élevage susceptibles de favoriser l'ingestion de sol. Le comportement d'exploration et un temps de séjour prolongé à l'extérieur sont des conditions nécessaires à l'augmentation de l'ingestion de matrices présentes sur le parcours. Des conditions climatiques favorables, une taille réduite de troupeau, certains types génétiques, le temps d'ouverture des trappes, qui favorisent le contact des animaux avec l'extérieur, sont donc des facteurs de risque (Kjaer et Isaksen 1998, Hegelund et al 2005). Selon Waegeneers et al (2009), la sensibilité des élevages de particuliers visà-vis des contaminants persistants de l'environnement proviendrait d'une consommation importante de sol. Elle serait due à un temps de séjour long sur les parcours, à la distribution de l'aliment à l'extérieur et à la dégradation du couvert végétal.

b) Disponibilité des contaminants présents dans le sol

Les études menées sur les aliments ne peuvent pas être directement utilisées pour estimer le transfert de contaminants ingérés avec le sol, les végétaux ou la pédofaune. En particulier, après leur dépôt, les contaminants interagissent avec les particules du sol et subissent un processus de maturation qui consiste principalement en des phénomènes d'adsorption mais également de piégeage dans les micropores du sol ou la matière organique (Reid et al 2000). Cette maturation contribue à la limitation de leur accessibilité dans le tube digestif. Van Eijkeren et al (2006) estiment que l'accessibilité des PCDD/F et PCB DL présents dans le sol est inférieure de $40 \%$ à celle des contaminants présents dans l'aliment. Mais cette valeur constitue une approximation car la nature et l'importance des interactions entre sol et contaminant dépendent des propriétés du sol et de celles de la molécule.

\section{Conclusion}

Les résultats des plans de contrôle et d'enquêtes montrent que les œufs commercialisés en Europe sont en grande majorité exempts de contaminants chimiques réglementés. Cette bonne qualité sanitaire est le résultat d'une réglementation stricte sur les aliments des animaux, sur l'usage des médicaments vétérinaires et additifs, et de son application par les acteurs de la filière. La mise en place des guides de bonnes pratiques en réponse au Paquet Hygiène devrait permettre de diminuer encore le taux de non-conformité et de garantir les produits vis-à-vis de ces risques. Les systèmes d'élevage qui ménagent 
un accès des animaux à l'extérieur sont plus fragiles vis-à-vis des contaminants de l'environnement pour lesquels l'exposition est difficilement contrôlable. Cependant, les cas de non-conformité demeurent rares pour les élevages com- merciaux. Les cas les plus préoccupants sont trouvés chez les particuliers. Cependant, afin de prévenir le risque dans ces élevages, il est important d'acquérir des connaissances sur l'impact des pratiques d'élevage sur l'ingestion de matrices environnementales et sur la disponibilité de ces contaminants lorsqu'ils sont présents dans des matrices telles que le sol.

\section{Références}

Anadon A., Martinez-Larranaga M.R. Fernandez-Cruz M.L., 1993. Considérations physiologiques et pharmacologiques en thérapeutique aviaire. Rev. Méd. Vét., 144, 745-757.

Arnold D., Somogyi A., 1986. Chloramphenicol residues in edible tissues of food animals. Proc. $2^{\text {nd }}$ World Congr. Foodborne Infection, Berlin, Germany, 832-836.

Blanc-Gonnet A., Bordet F., Deceuninck Y., Roudaut B., 2009. Bilan des résultats des plans de contrôle 2007 de substances chimiques chez les animaux d'exploitation et dans leurs produits. Bull. Epidémiologique, 32, 14-16.

Brugère H., 1992. Pharmacologie chez les oiseaux. In: Manuel de pathologie aviaire, Brugère-Picoux J., Silim A. (Eds). ENVA, Maisons-Alfort, France, 355-363.

Cannavan A., Ball G., Kennedy G., 2000. Nicarbazin contamination in feeds as a cause of residues in eggs. Food Add. Contam., 17, 829836.

Décision 97/747/CE de la Commission du 27 octobre 1997 fixant les niveaux et fréquences de prélèvement d'échantillons prévus par la directive 96/23/CE du Conseil en vue de la recherche de certaines substances et de leurs résidus dans certains produits animaux. J. Off. Commun. Eur., L303, 06.11.1997, 12-15.

Décision 2002/657/CE de la Commission portant sur les modalités d'application de la directive 96/23/CE du Conseil en ce qui concerne les performances des méthodes d'analyse et l'interprétation des résultats. 2002. J. Off. Commun. Eur., L221, 17.08.2002, 8-36.

De Vries M., Kwakkel R.P., Kijlstra A., 2006. Dioxins in organic eggs: a review. NJAS, 54, 207-2006.

Directive 86/363/CEE du Conseil, du 24 juillet 1986 concernant la fixation de teneurs maximales pour les résidus de pesticides sur et dans les denrées alimentaires d'origine animale. J.Off. Commun. Eur., L363, 7.08.1986, 1-17.

Directive 96/23/CE du Conseil du 29 avril 1996 relative aux mesures de contrôle à mettre en œuvre à l'égard de certaines substances et de leurs résidus dans les animaux vivants et leurs produits et abrogeant les directives 85/358/CEE et 86/469/CEE et les décisions 89/187/CEE et 91/664/CEE. J. Off. Commun. Eur., L125, 23.05.1996, 10-32.

Directive 2001/82/CE du Parlement européen et du Conseil, du 6 novembre 2001, instituant un code communautaire relatif aux médicaments vétérinaires. J. Off. Commun. Eur., L311, 28.11.2004, 1-66.

Directive 2002/32/CE du Parlement Européen et du Conseil du 7 mai 2002 sur les substances indésirables dans les aliments pour animaux. J. Off. Commun. Eur., L140, 30.05.2002, 10-21.
Donoghue D.J, Schenck F.J., Hairston H.,Podhorniak, L.V., 1997. Modeling drug residue uptake by eggs: Evidence of a consistent daily pattern of contaminant transfer into developing preovulatory yolks. J. Food Prot., 60, 1251-1255.

Dubreil-Chéneau E., Bessiral M., Roudaut B., Verdon E. Sanders P., 2009. Validation of a multi-residue liquid chromatography-tandem mass spectrometry confirmatory method for 10 anticoccidials in eggs according to commission decision 2002/657/EC. J. Chromatogr. A, 1216, 8149-8157.

EFSA, 2005. Opinion of the scientific panel on contaminants in the food chain on a request from the Commission related to the presence on non dioxin-like polychlorinated biphenyls (PCB) in feed and food. Question $\mathrm{N}^{\circ}$ EFSA-Q2003-114. European Food Safety Authority, The EFSA J., 284, 1-137.

Fournier A., Feidt C., Martin O., Travel A., Jondreville C., 2009. Elaboration d'un modèle de transfert des polluants organiques persistants du sol vers l'œuf de poule : un outil pour sécuriser les systèmes de production avicole.

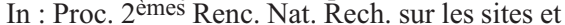
sols pollués, 20-21 octobre, Paris, France, 4p.

Fournier F., Feidt C., Dziurla M.A., Grandclaudon C., Jondreville C., 2010. Transfer kinetics to egg yolk and mode-ling residue recovered in yolk of rea-dily metabolized molecules: Polycyclic Aromatic Hydrocarbons orally administered to laying hens. Chemosphere, 78, 10041010

Harnly M.E., Petreas M.X., Flattery J., Goldman L.R., 2000. Polychlorinated dibenzop-dioxin and polychlorinated dibenzofuran contamination in soil and home-produced chicken eggs near pentachlorophenol sources. Env. Sci. Technol., 34, 1143-1149.

Hegelund L., Sørensen J. T., Kjær J. B., Kristensen I. S., 2005. Use of the range area in organic egg production systems: Effect of climatic factors, flock size, age and artificial cover. Brit. Poult. Sci., 46, 1-8.

Horsted K., Hermansen J.E., Ranvig H., 2007. Crop content in nutrient-restricted versus non-restricted organic laying hens with access to different forage vegetations. Brit. Poult. Sci., 48, 177-184.

Kan C.A., Petz M., 2000. Residues of veterinary drugs in eggs and their distribution between yolk and white. J. Agric. Food Chem., 48, 6397-6403.

Kan C.A., Meijer G.A.L., 2007. The risk of contamination of food with toxic substances present in animal feed. Anim. Feed Sci. Technol., 133, 84-108.

Kan K., 2005. Chemical residues in poultry and eggs produced in free-range or organic systems. Proc. XIth Eur. Symp. Quality of egg and egg products. Doorwerth, 23-26 May, The Netherlands, 210, $8 \mathrm{p}$.
Kjaer J.B., Isaksen P.K., 1998. Individual use of the free range area by laying hens and effects of genetic strain. Proc. $32^{\text {nd }}$ Congr. Int. Soc. Applied Ethology, Veissier, I., Boissy A. (Eds), 21-25 Juillet, Clermond-Ferrand, France, 88.

Leeman W.R., Van Den Berg K.J., Houben G.F., 2007. Transfer of chemicals from feed to animal products: The use of transfer factors in risk assessment. Food Add. Contam., 24, 1-13.

MacLachlan D.J., 2008. Transfer of fat-soluble pesticides from contaminated feed to poultry tissues and eggs. Brit. Poult. Sci., 49, 290298.

Mortier L., Huet A.C., Daeseleire E., Huyghebaert G., Fodey T., Elliott C., Delahaut P., Van Peteghem C., 2005. Deposition and depletion of five anticoccidials in eggs. J. Agric. Food Chem., 53, 7142-7149.

Opdycke J.C., Menzer R.E., 1984. Pharmacokinetics of diflubenzuron in two types of chickens. J. Toxicol. Env.. Health, 134, 721733.

Pussemier L., Mohimont L., Huyghebaert A., Goeyens L., 2004. Enhanced levels of dioxins in eggs from free range hens; A fast evaluation approach. Talanta, 63, 1273-1276.

Règlement (CEE) n²377/90 du Conseil du 26 juin 1990 établissant une procédure communautaire pour la fixation des limites maximales de résidus de médicaments vétérinaires dans les aliments d'origine animale. J. Off. Commun. Eur., L224, 18.08.1990, 1-8.

Règlement (CE) $n^{\circ} 1831 / 2003$ de la Commission du 22 septembre 2003 relatif aux additifs destinés à l'alimentation des animaux. J. Off. Commun. Eur., L268, 18.10.2003, 2943.

Règlement (CE) n³96/2005 du Parlement européen et du Conseil du 23 février 2005 concernant les limites maximales applicables aux résidus de pesticides présents dans ou sur les denrées alimentaires et les aliments pour animaux d'origine végétale et animale et modifiant la directive 91/414/CEE du Conseil. J. Off. Commun. Eur., L70, 16.03.2005, 1-16

Règlement CE n $1881 / 2006$ de la Commission du 19 décembre 2006 portant fixation de teneurs maximales pour certains contaminants dans les denrées alimentaires. J. Off. Commun. Eur., L364, 20.12.2006, 5-24.

Règlement CE n ${ }^{\circ} 124 / 2009$ de la Commission du 10 février 2009 établissant des valeurs maximales pour la présence dans les denrées alimentaires de coccidiostatiques ou d'histomonostatiques résultant du transfert inévitable de ces substances vers des aliments pour animaux non-cibles. J. Off. Commun. Eur., L40, 11.02.2009, 7-11.

Règlement CE $n^{\circ} 470 / 2009$ du Parlement européen et du Conseil du 6 mai 2009 établissant des procédures communautaires pour la 
fixation des limites de résidus des substances pharmacologiquement actives dans les aliments d'origine animale, abrogeant le règlement (CEE) no 2377/90 du Conseil et modifiant la directive 2001/82/CE du Parlement européen et du Conseil et le règlement (CE) no 726/2004 du Parlement européen et du Conseil. J. Off. Commun. Eur., L152, 16.06.2009, 1122.

Reid B.J., Jones K.C., Semple K.T., 2000. Bioavailability of persistent organic pollutants in soils and sediments: a perspective on mechanisms, consequences and assessment. Env.. Pollut., 108, 103-112.

Renwick A.G., 2001. Toxicokinetics: Pharmacokinetics in toxicology. In: Principles and methods of toxicology. Hayes A.W. (Eds). Taylor Francis, Philadelphia, PA, USA, 137-191.

Roudaut B., 1997. Antibiothérapie chez la poule pondeuse. Etude des résidus d'antibiotiques dans les œufs. Thèse de doctorat. Institut Polytechnique de Toulouse, France, 181p.

Roudaut B., 1998. Elimination of oxolinic acid in eggs after oral treatment of laying hens. Brit. Poult. Sci., 39, 47-52.

Roudaut B., Garnier M., 2002. Sulphonamide residues in eggs following drug administration via the drinking water. Food. Addit. Contam., 19, 373-378.

Samouris G., Tsoukali-Papadopoulou H., Nathanael B., Mirtsou-Fidany V., 1998. Chloramphenicol residues in albumenn and yolk of hen's eggs after experimental administration. Arch. Gefluegelk., 62, 83-85.

Schmid P., Gujer E., Dengen S., Zenneg M. Kuchen A., Wäthrich C., 2002. Levels of polychlorinated dibenzo-p-dioxins and dibenzofurans in food of animal origin. The Swiss dioxin monitoring program. J. Agric. Food Chem., 50, 7482-7487.

Schoeters G., Hoogenboom R., 2006 Contamination of free-range chicken eggs with dioxins and dioxin-like polychlorinated biphenyls. Mol. Nutr. Food Res., 50, 908-914.

Schuler F., Schmid P., Schlatter C., 1997. The transfer of polychlorinated dibenzop-dioxins and dibenzofurans from soil into eggs of foraging chicken. Chemosphere, 34 711-718.

Stephens R.D., Petreas M.X., Hayward D.G. 1995. Biotransfer and bioaccumulation of dioxins and furans from soil: Chickens as a model for foraging animals. Sci. Total Env., $175,253-273$.

Thébault A., 2005. Analyse des déterminants de la contamination en dioxines et furanes (PCB non compris) des oeufs issus d'élevages de volailles en plein air de particuliers. Note technique AQR/ATH/2005-203, AFSSA, 27 septembre 2005, 17p. http://www.editionsduboisbaudry.fr/docs/av/pdf/2006-06/2005-0927 Afssa dioxine.pdf

Travel A., Jondreville C. Guinvarch J., Chabault M., Lubac S., Feidt C., Marchand P.
Bonnard R., Le Bouquin-Neveu S., Allain V., Thébault A., Gonnier V., Nys Y., 2008. La filière fait le point sur le risque de transfert de polluants organiques persistants vers les œufs. Tema, 6, 11-19.

Travel A., Guinvarch J., Rouxel S., Homo N., Thébault A., Marchand P., Chabault M., Lubac S., Marze L., Nys Y., S. Le Bouquin, 2009. Les polluants organiques persistants dans les œufs: facteurs de risque en l'élevage.

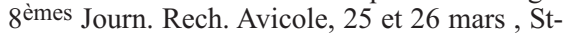
Malo, France, $4 \mathrm{p}$.

Van Eijkeren J.C.H., Zeilmaker M.J., Kan C.A., Traag W.A., Hoogenboom L.A.P.,2006 A toxicokinetic model for the carry-over of dioxins and PCBs from feed and soil to eggs. Food Addit. Contam., 23, 509-517.

Van Overmeire I., Pussemier L., Waegeneers N., Hanot V., Windal I., Boxus L., Covaci A., Eppe G., Scippo M. L., Sioen I., Bilau M., Gellynck X., De Steur H., Tangni, E.K., Goeyens L., 2009. Assessment of the chemical contamination in home-produced eggs in Belgium: General overview of the CONTEGG study. Sci. Tot. Env., 407, 4403-4410.

Waegeneers N., De Steur H., De Temmerman L., Van Steenwinkel S., Gellynck X., Viaene J., 2009. Transfer of soil contaminants to home-produced eggs and preventive measures to reduce contamination. Sci. Tot. Env., 407, 4438-4446.

\section{Résumé}

Le présent article fait le point sur les contaminants chimiques organiques potentiellement présents dans les oufs de poules pondeuses. Ils ont pour origine soit des médicaments vétérinaires, des additifs introduits dans les aliments, des pesticides utilisés pour la production des matières premières entrant dans l'alimentation des animaux ou encore des polluants rémanents de l'environnement. Les médicaments vétérinaires et additifs alimentaires font l'objet d'études strictes de transfert avant d'être autorisés et les résultats d'enquêtes ou de contrôles officiels montrent que les limites maximales de résidus sont très rarement dépassées dans les œufs. Les nonconformités ont en général pour origine une utilisation inadéquate de la molécule, ou des contaminations croisées lors de la fabrication des aliments, notamment pour les anticoccidiens. Les pesticides utilisés pour la production de matières premières entrant dans l'alimentation des animaux font également l'objet d'une étude d'évaluation du risque de transfert avant leur autorisation. L'exposition des animaux aux contaminants de l'environnement est plus difficilement contrôlable, notamment dans les élevages en plein air, et des dépassements importants des teneurs maximales autorisées pour les dioxines, furanes ou polychlorobiphényles sont parfois rencontrés dans ce type d'élevage. Les cas les plus préoccupants émanent d'élevages de particuliers, dans lesquels certaines pratiques semblent favoriser l'ingestion de matrices, notamment de sol, en quantités importantes. Afin de prévenir le risque de transfert de ces contaminants, il est important d'évaluer l'impact de ces pratiques sur l'exposition des animaux et de quantifier l'impact de la matrice sol sur le transfert des contaminants ingérés vers l'œuf.

\section{Abstract}

\section{Organic chemical contaminants in hens egg: regulatory context, modes and risk of transfer}

The current paper reviews current knowledge on the organic chemical contaminants that may be present in hen eggs. They originate either from veterinary drugs or feed additives, from pesticides used for cereals production or from persistent pollutants of the environment. Carry-over studies are carried out before veterinary drugs and additives are allowed. For these compounds, official surveys reveal concentrations that are barely over the maximal residues limits. Excessive concentrations may be detected only in cases of inappropriate use of veterinary drugs or of cross contamination during feed processing, especially for coccidiostats. Carry-over experiments are also conducted before allowing pesticides to be used for cereal production. Effective control against environmental contaminants for animals raised outdoors is not easy. In fact, concentrations of dioxins, furans and polychlorobiphenyls exceeding the maximum concentrations permitted in eggs have been rarely recorded. The worst cases arise in home-produced eggs, probably as a result of practices that stimulate ingestion of environmental matrices, especially soil. In order to prevent the risk of the presence of such contaminants in eggs, factors affecting soil ingestion in animals raised outdoors should be better understood, and the impact of soil on the carry-over of ingested contaminants to eggs should be quantified.

JONDREVILLE C., FOURNIER A., TRAVEL A., FEIDT C., ROUDAUT B., 2010. Contaminants chimiques organiques des œufs de poule pondeuse : aspects réglementaires, modalités et risques de transfert. In : Numéro Spécial, Qualité de l'œuf. Nys Y. (Ed). Inra Prod. Anim., 23, 205-214. 
